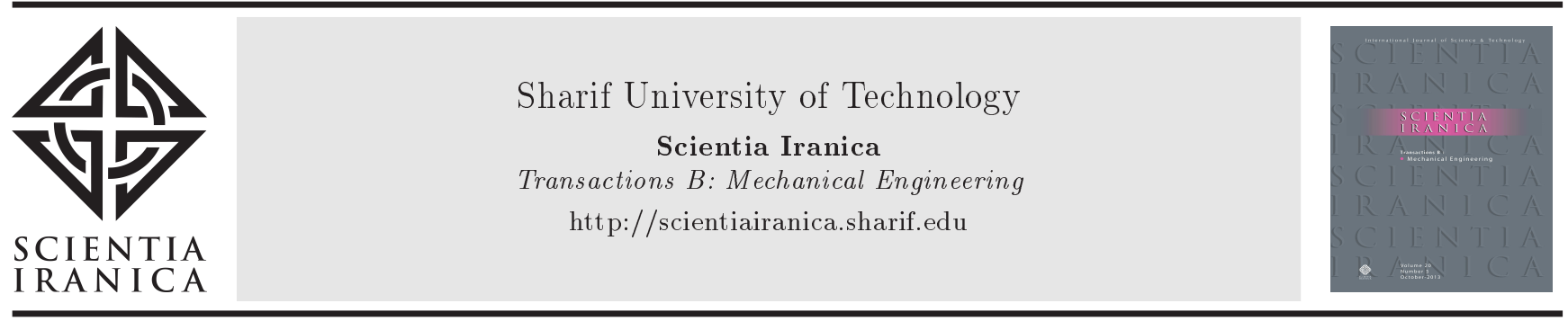

Research Note

\title{
Optimal tuner selection using Kalman filter for a real-time modular gas turbine model
}

\author{
R. Sheikhbahaei, G. Vossughi, and A. Alasty* \\ Department of Mechanical Engineering, Sharif University of Technology (SUT), Tehran, P.O. Box 11155-9567, Iran.
}

Received 9 October 2017; received in revised form 1 August 2018; accepted 26 January 2019

\author{
KEYWORDS \\ Gas turbine; \\ Dynamic modeling; \\ Modular modeling; \\ Model tuning; \\ Optimal tuner \\ selection; \\ Underdetermined \\ estimation; \\ Kalman filter.
}

\begin{abstract}
In this study, a real-time flexible modular modeling approach to simulating the dynamic behavior of gas turbine engines based on nonlinear thermodynamic and dynamic laws is addressed. The introduced model, which is developed in the Matlab-Simulink environment, is an object-oriented high-speed real-time computer model and is capable of simulating the dynamic behavior of a broad group of gas turbine engines due to its modular structure. Moreover, a Kalman filter-based model tuning procedure is applied to decrease the modeling errors. Modeling errors are defined as the mismatch between simulation results and available experimental data. This tuning procedure is an underdetermined estimation problem, where there are more tuning parameters than available measured data. Here, an innovative approach to producing a tuning parameter vector is introduced. This approach is based on seeking an optimal initial value for the Kalman filter tuning procedure. Three simulation studies are carried out in this paper to demonstrate the advantages, capabilities, and performance of the proposed scheme. Furthermore, simulation results are compared with manufacturers' published data and with the experimental results gathered in either turbo-generator or turbo-compressor applications. The computational time requirement of the model is discussed at the end of the paper.

(C) 2020 Sharif University of Technology. All rights reserved.
\end{abstract}

\section{Introduction}

Gas turbines enjoy widespread applications in jet engines, turbo-generators, marine industries, and natural gas transmission over long distances. These engines generally consist of a multi-stage axial compressor, a combustion chamber, and an axial turbine and operate in the Brayton cycle [1]. Based on the number of shafts, gas turbines are categorized into two distinct types [1] (Figure 1). Due to its diverse applications, gas tur-

*. Corresponding author. Tel.: +982166165504 E-mail addresses: rezashb@mech.sharif.ir (R. Sheikhbahaei); vossough@sharif.edu (G. Vossughi); aalasti@sharif.edu (A. Alasty).

doi: $10.24200 /$ sci.2019.5341.1218 bine modeling is essential in the design, development, control, and condition monitoring of such a complex system.

During the last few decades, based on various applications and limitations, several models have been introduced for gas turbines in either frequency domain [2] or time domain [3]. These models are presented in three general approaches: black-box, graybox, and white-box. In the first approach, the model is obtained using some paired input-output data [47]. In doing so, the internal structure of the system is neglected. Gray-box modeling is the second approach, in which a specific physical structure is considered and each part/subsystem is represented by some identified or empirical models (for example, see $[8,9]$ ). The third approach, i.e., white-box modeling, is based on the governing dynamic and thermodynamic laws 


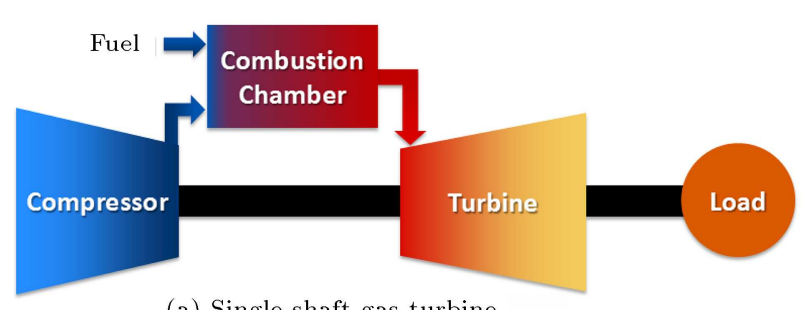

(a) Single shaft gas turbine

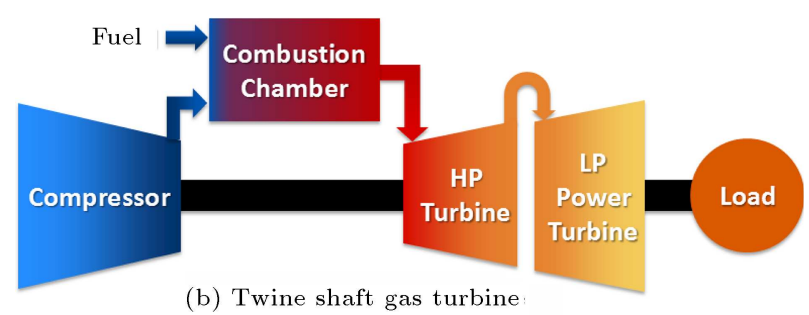

Figure 1. Single-shaft and twin-shaft gas turbines.

that make it more complex, yet more reliable. In addition, the internal structure has a real physical interpretation that can be rearranged and tuned for almost all gas turbines. Thermodynamic laws that are often involved in gas turbines modeling [10] consist of mass, energy, and momentum conservation laws. Due to their inherent accuracy, these models are the best choice for simulation purposes and data generation.

Component Level Models (CLM) constitute a group of gas turbine white-box models (for example, see [11-15]), in which a complete dynamic model is obtained by arranging separately developed component models beside each other. However, some data are transferred among these components as feed-forward or feedback signals. CLMs are also known as modular models. In a comprehensive manner, in some gas turbine modular modeling papers like [11], modeling details have been left out. In some other papers such as $[13,14]$, the cooling effect was not considered in turbine expansion; in studies such as $[15,16]$, the volume dynamic effect was not completely modeled as in this paper. Besides modeling, model tuning should also be emphasized as a vital step in obtaining a valid model.

Model tuning is achieved through parameter estimation $[17,18]$ and fine-tuning of Tuning Parameters (TPs) embedded in the model to tackle the uncertainties (for example, see $[19,20]$, on gas turbine model tuning). The most useful parameter estimation method is the Extended Kalman Filter (EKF) [21], which can estimate TPs in a real-time fashion. The EKF has a unique solution for an observable system with enough measured data, while the number of measurements in a gas turbine is typically lower than that of uncertain TPs. Thus, it is an underdetermined estimation problem with no unique solution. An approach to countering this problem is to estimate a subset of TPs detected that are more effective in available measured data. To this end, the subset vector, $s$, can be produced as a linear combination of TP vector, $b$, as $s=X b$. In [22], the transformation matrix, $X$, was selected by applying singular value decomposition, whereas an optimization routine was proposed for the same purpose in [23]. In [22], the elements of the transformation matrix comprised zeros and ones; therefore, some TPs were left unchanged, which could considerably decrease the modeling error. In [23], the transformation matrix was iteratively optimized to minimize the modeling error. At each iteration, the transformation matrix, $X$, was updated as the optimization variable, the vector $s$ was subsequently computed by the use of the EKF, and the corresponding modeling error was calculated using pseudo-inverse of $X$. Now, the question is: What will happen if the EKF is used for an underdetermined problem? In response, the solution depends on the initial vector of TPs. In this case, the EKF initial vector could be the optimization variable. This approach is the main contribution of this paper.

In this paper, a detailed approach to modular modeling and dynamic simulation of gas turbine engines is introduced, which is easy to understand, straightforward to rebuild, applicable to various gas turbine architectures, rapid to respond, and sufficiently accurate. Afterward, an innovative model tuning procedure is discussed, in which an optimization routine including the EKF is utilized to adjust the vector of TPs embedded in the model. Finally, a comprehensive case study on a twin-shaft gas turbine is presented for model validation.

The rest of this paper is organized as follows. The gas turbine employed in the case study is introduced in Section 2. In Section 3, the modeling procedure and the relevant equations are presented and, in the sequel, the model tuning and the optimal tuner selection are discussed. Simulation results and model validation are addressed in Section 5; finally, the concluding remarks are presented in Section 6.

\section{Gas turbine description}

An industrial twin-shaft compact light-weight gas turbine manufactured by Siemens Industrial Turbomachinery is examined as the case study to evaluate and validate the developed model (discussed in Section 3). This gas turbine, namely SGT-600, is employed in both fields of power generation and mechanical drive applications. Its mechanical structure consists of a ten-stage axial compressor rotating on a common shaft with a two-stage turbine. Variable Inlet Guide Vanes (VIGVs) are located in the first two stages of the compressor to adjust the air mass flow rate. The outlet flow of the first turbine to the second two-stage power turbine rotating on a separate shaft is conducted.

The control system of this gas turbine is a 
Table 1. Nominal design point specifications of SGT-600 [24].

\begin{tabular}{lc}
\hline \multicolumn{1}{c}{ Parameter } & Value \\
\hline Output power (MW) & 24.77 \\
Thermal efficiency (\%) & 34.2 \\
Compressor pressure ratio & 14 \\
Exhaust gas temperature $(\mathrm{K})$ & 816 \\
Exhaust mass flow rate $(\mathrm{kg} / \mathrm{s})$ & 80.4 \\
Gas generator rotational speed $(\mathrm{rpm})$ & 9705 \\
Power turbine rotational speed $(\mathrm{rpm})$ & 7700 \\
\hline
\end{tabular}

combination of several limiters besides a ProportionalIntegral (PI) stabilizing controller. Limiters are considered to keep the gas turbine operating point in the predefined safe and stable zone of operation. The PI controller is also designed to adjust fuel flow, as the main control variable, to stabilize the output variables such as the power turbine's rotational speed or output power. The other control variable is the position of VIGVs controlled in an open-loop manner, such that the VIGV position is just a function of the gas generator's rotational speed. The specifications of nominal design point performance are listed in Table 1 [24], and the explanation of the control system is available in [25].

\section{Modeling}

The developed mathematical model is a CLM with a modular representation of gas turbine components. A detailed explanation of equations is discussed in this section. The iterative nature of calculations employed for dynamic modeling does not deteriorate the model performance and the convergence is achieved sufficiently rapidly. Furthermore, changes are easily made to such a modular model, and the behavior of a broad group of gas turbines can be simulated by the edition, addition, or deletion of components.

\subsection{Model setup}

The model is developed in Matlab-Simulink as an object-oriented tool for modular modeling. Almost all the components are modeled by a combination of two blocks. One block is designed to simulate the static behavior of the component as a volumeless element, and the other one, namely "plenum", is employed to consider the unsteady mass and energy balance of the component in order to represent its dynamic behavior. Commonly, temperature, pressure, and molar fractions of inlet and outlet flows of each component are considered as the input and output variables, respectively. The other input variable is the outlet mass flow rate of the next component applied as a feedback (see Figure 2). Furthermore, to satisfy the

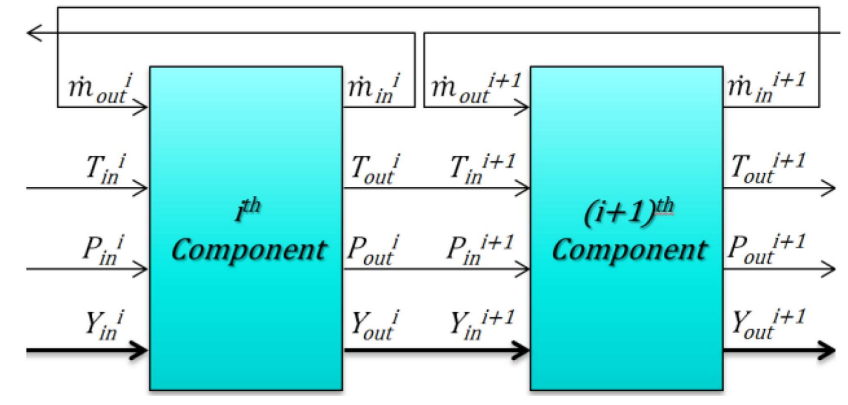

Figure 2. Common I/O in gas path components of the model.

governing equations of rotor dynamics, blocks related to the rotating shafts treat the rotating speed and torque as Input/Output (I/O).

As presented in Figure 3, the main modules of a twin-shaft gas turbine model consist of a compressor, a combustion chamber, a gas generator turbine, a power turbine, shafts, and governor subsystems, each of which involves a set of sub-modules.

\subsection{Thermodynamic properties}

The instantaneous thermodynamic properties of gaseous stream depend on its instantaneous temperature and composition. Gas flow composition is taken into account by applying its chemical components' molar fraction, undergoing changes in the combustion process and, also, in mixing with other previously split streams such as cooling air. The primary flow entering the gas turbine is assumed to be fresh air with the composition listed in Table 2 [26].

As argued in [27], $\mathrm{C}_{n} \mathrm{H}_{2(n+1)}$ can be considered as the structural formula of alkanes. Due to the swirling of excessive air involved in the reactions taking place in the combustor, a complete combustion process is assumed as in Reaction (1). Subsequently, the molar fractions of the main stream after combustion are obtained through Eqs. (2):

$$
\begin{aligned}
& \mathrm{C}_{n} \mathrm{H}_{2(n+1)}+\frac{3 n+1}{2} \mathrm{O}_{2} \rightarrow n \mathrm{CO}_{2}+(n+1) \mathrm{H}_{2} \mathrm{O}, \\
& y_{\mathrm{N}_{2}}^{2}=\frac{y_{\mathrm{N}_{2}}^{1}}{1+\left(\frac{n+1}{2}\right) x_{f}},
\end{aligned}
$$

Table 2. Fresh air composition [26].

\begin{tabular}{cc}
\hline Air composition & Molar fraction (\%) \\
\hline $\mathrm{N}_{2}$ & 78.076 \\
$\mathrm{O}_{2}$ & 20.945 \\
$\mathrm{Ar}$ & 0.934 \\
$\mathrm{CO}_{2}$ & 0.035 \\
$\mathrm{H}_{2} \mathrm{O}$ & 0.010 \\
\hline
\end{tabular}




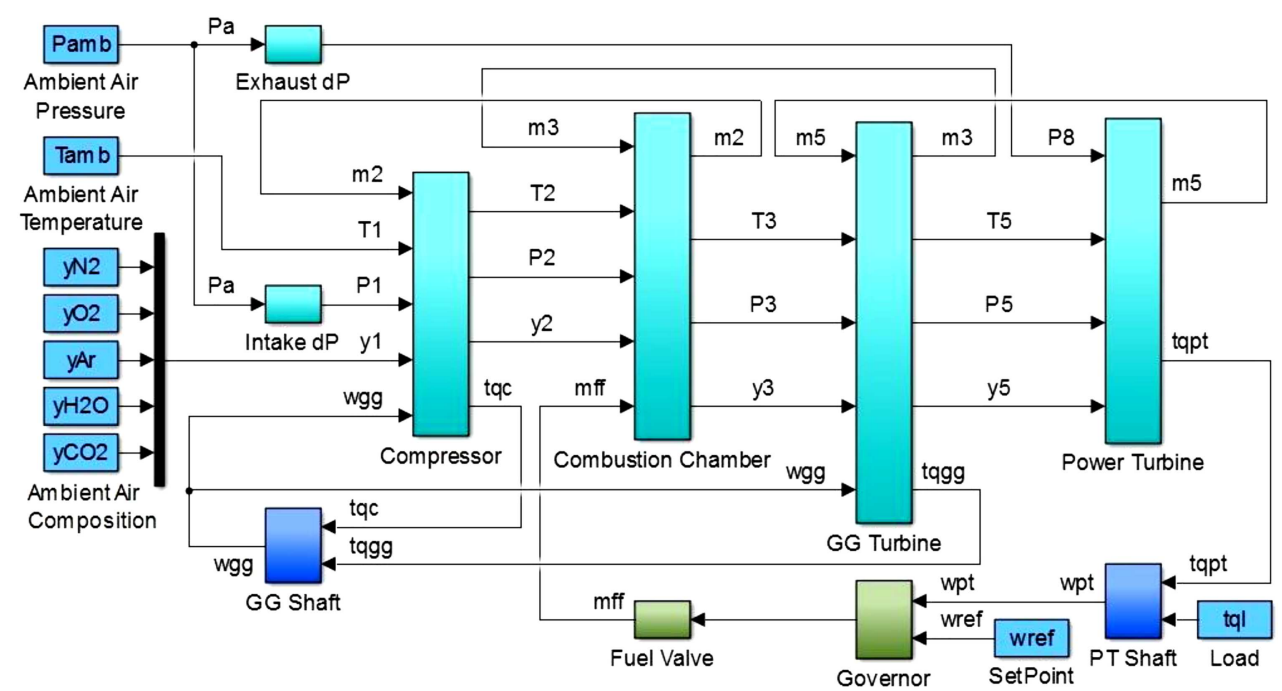

Figure 3. Main modules of a twin-shaft gas turbine model.

$$
\begin{aligned}
& y_{\mathrm{Ar}}^{2}=\frac{y_{\mathrm{Ar}}^{1}}{1+\left(\frac{n+1}{2}\right) x_{f}}, \\
& y_{\mathrm{O}_{2}}^{2}=\frac{y_{\mathrm{O}_{2}}^{1}-\left(\frac{3 n+1}{2}\right) x_{f}}{1+\left(\frac{n+1}{2}\right) x_{f}}, \\
& y_{\mathrm{CO}_{2}}^{2}=\frac{y_{\mathrm{CO}_{2}}^{1}+n x_{f}}{1+\left(\frac{n+1}{2}\right) x_{f}}, \\
& y_{\mathrm{H}_{2} \mathrm{O}}^{2}=\frac{y_{\mathrm{H}_{2} \mathrm{O}}^{1}+(n+1) x_{f}}{1+\left(\frac{n+1}{2}\right) x_{f}},
\end{aligned}
$$

where $x_{f}$ is the molar flow rate ratio of the involved alkane to the main stream and is computed in the following:

$$
x_{f}=\frac{\dot{m}_{\mathrm{C}_{n} \mathrm{H}_{2(n+1)}} / M_{\mathrm{C}_{n} \mathrm{H}_{2(n+1)}}}{\dot{m}_{a} / M_{a}} .
$$

For different fuel compositions (with several alkanes), new molar fractions after combustion can be obtained through a partially modified version of Eqs. (2). Molar fractions after the mixing process can be obtained using Eq. (4), in which subscript indices $a_{1}$ and $a_{2}$ represent two gaseous streams before mixing.

$$
\begin{aligned}
& y_{i}^{2}=\frac{\left(y_{i}^{1}\right)_{a_{1}}\left(\frac{\dot{m}_{a_{1}}}{M_{a_{1}}}\right)+\left(y_{i}^{1}\right)_{a_{2}}\left(\frac{\dot{m}_{a_{2}}}{M_{a_{2}}}\right)}{\frac{\dot{m}_{a_{1}}}{M_{a_{1}}}+\frac{\dot{m}_{a_{2}}}{M_{a_{2}}}}, \\
& i=\mathrm{N}_{2}, \mathrm{Ar}, \mathrm{O}_{2}, \mathrm{CO}_{2}, \mathrm{H}_{2} \mathrm{O} .
\end{aligned}
$$

To evaluate the thermodynamic properties of the stream, i.e., specific heat and total enthalpy, the following polynomial laws of total temperature [28] are used:

$$
\begin{aligned}
C_{p}(T) & =R \sum_{i} \frac{y_{i} C_{p_{i}}(T)}{R_{i}}, \\
i & =\mathrm{N}_{2}, \mathrm{Ar}, \mathrm{O}_{2}, \mathrm{CO}_{2}, \mathrm{H}_{2} \mathrm{O}, \\
C_{p_{i}}(T) & =R_{i} \sum_{j=1}^{5} a_{i, j} T^{(j-1)}, \\
h(T) & =R \sum_{i} \frac{y_{i} h_{i}(T)}{R_{i}}, \\
i & =\mathrm{N}_{2}, \mathrm{Ar}_{,} \mathrm{O}_{2}, \mathrm{CO}_{2}, \mathrm{H}_{2} \mathrm{O}, \\
h_{i}(T) & =R_{i}\left(a_{i, 6}+\sum_{j=1}^{5} \frac{a_{i, j}}{j} T^{j}\right) .
\end{aligned}
$$

By considering a different composition for the gas turbine inlet air, it is possible to study the influence of the ambient air humidity and, also, water or steam injection on the overall performance of a gas turbine.

\subsection{Plenum}

The unsteady mass and energy balance of each component is modeled considering an adiabatic capacity besides its main block. A plenum is placed in the blocks of the compressor, combustion chamber, and turbine stages. Mass and energy conservation laws for a one-dimensional flow in the plenum are respectively as follows:

$$
\begin{aligned}
\frac{d m}{d t} & =\dot{m}_{\text {in }}-\dot{m}_{\text {out }}, \\
\frac{d U}{d t} & =(\dot{m} h)_{\text {in }}-(\dot{m} h)_{\text {out }} .
\end{aligned}
$$


The heat energy flows and the work terms are not taken into account. Considering that $U=m C_{v} T$, Eq. (8) is reformulated as follows:

$$
\frac{d U}{d t}=C_{\nu} T \frac{d m}{d t}+m C_{\nu} \frac{d T}{d t}=(\dot{m} h)_{\text {in }}-(\dot{m} h)_{\text {out }}
$$

Since $m=\rho \nu_{p}$ and $\gamma=C_{p} / C_{\nu}$, the combination of Eqs. (7) and (9) results in the following:

$$
\frac{d T}{d t}=\frac{\gamma}{\rho C_{p} \nu_{p}}\left((\dot{m} h)_{\text {in }}-(\dot{m} h)_{\text {out }}\right)+\frac{T}{\rho \nu_{p}}\left(\dot{m}_{\text {out }}-\dot{m}_{\text {in }}\right) \text { (10) }
$$

The ideal gas equation, i.e., $P=\rho R T$, and the density definition, i.e., $m=\rho \nu_{p}$, are used along with Eqs. (7) and (10) to determine another state equation governing the total pressure as follows:

$$
\frac{d P}{d t}=\rho R \frac{d T}{d t}+\frac{R T}{\nu_{p}} \frac{d m}{d t}=\frac{\gamma R}{C_{p} \nu_{p}}\left((\dot{m} h)_{\text {in }}-(\dot{m} h)_{\text {out }}\right) \text { (1 }
$$

\subsection{Compressor}

The compressor block is composed of two sub-blocks. The first one implements the steady state compressor characteristic curves and depicts the quasi-steady behavior of the compressor. A plenum is placed as the second sub-block, which calculates output temperature and pressure. The characteristic curves, which are developed by computational fluid dynamics methods for a finite number of operating points, are applied to calculate the mass flow rate and isentropic efficiency of the compressor as a function of pressure ratio and corrected rotational speed as Eq. (12). Implementing these curves requires numerical interpolation, as discussed in Subsection 3.9.

$$
\left(\dot{m}_{c}, \eta_{c}\right)=f\left(T_{\text {in }}, P_{\text {in }}, P_{\text {out }}, \omega_{c}\right) .
$$

The required mechanical torque of the compressor $\left(q_{c}\right)$ is calculated as a function of its rotational speed $\left(\omega_{c}\right)$ as follows [10]:

$$
q_{c}=\frac{\dot{m}_{c}\left(h_{o u t}-h_{\text {in }}\right)}{\omega_{c}} .
$$

The outlet temperature of the compressor before including the dynamic effects in plenum block is calculated [10] as follows:

$$
T_{c}=T_{\text {in }}\left[1+\frac{1}{\eta_{c}}\left({\frac{P_{\text {out }}}{P_{\text {in }}}}^{(\bar{\gamma}-1) / \bar{\gamma}}-1\right)\right],
$$

where $\bar{\gamma}$ is evaluated at $\bar{T}=\left(T_{i n}+T_{c}\right) / 2$.

\subsection{Combustion chamber}

The block of a combustion chamber also contains a plenum along with two other sub-blocks, whichare located to calculate the mass flow rate and the combustion temperature rise. The mass flow rate is

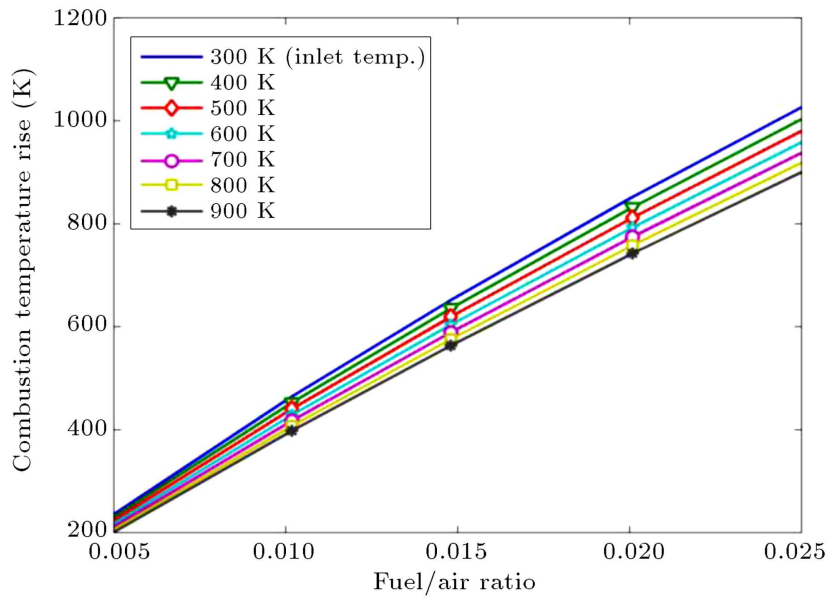

Figure 4. Combustion temperature rise versus the fuel-to-air ratio and inlet air temperature.

calculated by Eq. (15), in which the coefficients, $a, b$ are dependent on the temperature and pressure of the inlet stream. Outlet mass flow rate is simply obtained by adding the fuel mass flow rate to $\dot{m}_{c c}$.

$$
\dot{m}_{c c}=a\left(1-\sqrt{1+b\left(P_{\text {in }}-P_{\text {out }}\right)}\right) .
$$

The temperature rise is calculated using lookup tables, available in references like [29], prepared for various typical fuel compositions. Figure 4 illustrates one sample plot of temperature rise versus the fuel-toair ratio and inlet flow temperature. These lookup tables are originated from the following energy balance equation considering the fact that the combustion process is adiabatic and has no work transfer [1].

$$
\begin{aligned}
& \left(\dot{m}_{i n}+\dot{m}_{f}\right) C_{P c c}\left(T_{c c}-298\right)+\dot{m}_{f} \Delta h_{25} \\
& \quad=\dot{m}_{i n} C_{P_{i n}}\left(T_{i n}-298\right)+\dot{m}_{f} C_{P_{f}}\left(T_{f}-298\right) .
\end{aligned}
$$

Parameter $\Delta h_{25}$ in Eq. (16) is the enthalpy of combustion at a reference temperature of $25^{\circ} \mathrm{C}$ with vaporized water in the reaction products (since products' temperature is undoubtedly above the dew point). Moreover, $C_{P_{c c}}\left(C_{P_{i n}}\right)$ is the mean specific heat of combustion products over the range of $298 \mathrm{~K}$ to $T_{c c}\left(T_{i n}\right)$. The second term on the right-hand side of this equation is negligible due to the small amount of $\dot{m}_{f}$ with respect to $\dot{m}_{i n}$, in addition to a small difference between $T_{f}$ and the reference temperature. Through this equation, a lookup table could be generated to estimate the temperature rise of the combustion process as follows:

$$
T_{c c}=T_{i n}+\Delta T\left(\frac{\dot{m}_{f}}{\dot{m}_{i n}}, T_{i n}\right) .
$$

\subsection{Turbine}

The stream flowing through the turbine stages is cooled in both stator vanes and rotor blades. To consider 
the cooling effects on gas turbine performance, the proposed scheme by El-Masri [30], which has been applied in $[15,16]$, is employed, in which enthalpy drop is found by mass and energy balances as follows:

$$
h_{\text {out }}=\frac{\dot{m}_{\text {in }} h_{\text {in }}+\dot{m}_{\text {cool }} h_{\text {cool }}}{\dot{m}_{\text {in }}+\dot{m}_{\text {cool }}} .
$$

Furthermore, mixing pressure loss is calculated using Eq. (19), where the coefficient $Y$ is the momentum loss parameter and is set to 0.15 for stator vanes and 0.2 for rotor blades [31]:

$$
\frac{\Delta P}{P}=Y \frac{\dot{m}_{\text {cool }}}{\dot{m}_{a}}\left(1+\frac{M_{a}}{M_{\text {cool }}}\right) \text {. }
$$

Thus, four sub-blocks are considered for modeling each turbine stage. Cooling effects, based on Eqs. (18) and (19), are taken into account for vanes and blades in the first and third blocks, respectively. In the second block, the steady state turbine characteristics are used to obtain the mass flow rate and isentropic efficiency of the turbine as a function of pressure ratio along with a corrected rotational speed as in Eq. (20). Numerical interpolation (discussed in Subsection 3.9) is also necessary for working with turbine characteristics. A plenum is considered as the fourth sub-block to take the dynamic behavior of outlet temperature and pressure into account.

$$
\left(\dot{m}_{t}, \eta_{t}\right)=g\left(T_{\text {in }}, P_{\text {in }}, P_{\text {out }}, \omega_{t}\right) \text {. }
$$

The torque generated in each stage is another output variable of this block, which is computed through the following equation [10]:

$$
q_{t}=\frac{\dot{m}_{t}\left(h_{\text {in }}-h_{\text {out }}\right)}{\omega_{t}}
$$

Moreover, the static outlet temperature of the turbine stage is obtained as follows [10]:

$$
T_{t}=T_{\text {in }}\left[1+\eta_{t}\left({\frac{P_{\text {out }}}{P_{\text {in }}}}^{(\bar{\gamma}-1) / \bar{\gamma}}-1\right)\right],
$$

where $\bar{\gamma}$ is evaluated at $\bar{T}=\left(T_{i n}+T_{t}\right) / 2$.

\subsection{Rotating shafts}

The dynamic behavior of each shaft is simply modeled by its angular momentum equilibrium. The angular acceleration of the shaft is proportional to the net torque delivered by considering loads and mechanical efficiency as follows:

$$
\frac{d \omega_{s}}{d t}=\frac{1}{J_{s}}\left(\eta_{m} \sum_{\substack{\text { Turbine } \\ \text { Stages }}} q_{t_{i}}-q_{c}-q_{l}\right),
$$

where $J_{s}$ represents the angular moment of inertia of the shaft. Furthermore, the effect of power losses and mechanical friction is considered via mechanical efficiency $\eta_{m}$.

\subsection{Governor}

Since this paper does not deal with the design and evaluation of the turbine control system, the governor is simply regarded as a PI stabilizing controller, in which fuel mass flow rate is adjusted to regulate the rotational speed of the power turbine. Furthermore, the fuel valve dynamics along with the fuel flow dynamics is simply simulated [32] as follows:

$$
\mathrm{T} . \mathrm{F} .=\frac{1}{0.07 s+1} \cdot \frac{1}{0.1 s+1},
$$

where $\frac{1}{0.07 s+1}$ is the valve positioner transfer function, and $\frac{1}{0.1 s+1}$ represents the fuel flow dynamics. The corresponding formulation is adopted from [32] while the constants, 0.07 and 0.1 , are derived from the time constants of the fuel system dynamics.

\subsection{Interpolation}

Compressor and turbine characteristic curves are available only for a finite number of operating points. For example, in an axial compressor, for each predefined corrected rotational speed, there are two curves that specify the relationships among pressure ratio, mass flow rate, and isentropic efficiency. For rotational speeds between the predefined values, interpolation is essential. $\beta$-lines interpolation method [16] is applied, in which an offline calculation routine is performed to map any predefined point of $(\omega, P R)$ to a new point of $(\alpha, \beta)$ in the transformed domain as follows:

$$
\begin{aligned}
& \alpha=\frac{\omega-\omega_{\min }}{\omega_{\max }-\omega_{\min }} \\
& \beta=\frac{P R-P R_{\min }(\omega)}{P R_{\max }(\omega)-P R_{\min }(\omega)},
\end{aligned}
$$

where, for example, for an axial air compressor, $P R_{\min }(\omega)$ and $P R_{\max }(\omega)$ are the minimum and maximum admissible pressure ratios (referred to as choking and surging lines) of the compressor, respectively, operating at the rotational speed, $\omega$.

In this way, all the available data within the compressor or turbine operating regime can be transformed into normalized three-dimensional Cartesian coordinates, in which linear interpolation could be possible. This method relies on the data generated by offline calculations and, therefore, is well suited for real-time interpolation.

The interpolation routine follows a sequence, where each step decrements the dimension of the data by one. The interpolation of rotational speeds, as the first variable, generates a $2 \mathrm{D}$ dataset for the desired speed. This step is illustrated in Figure 5, in which the new constant speed line (red marked line) is interpolated between two predefined speed lines. The next step involves PR interpolation, in which a $1 \mathrm{D}$ dataset 


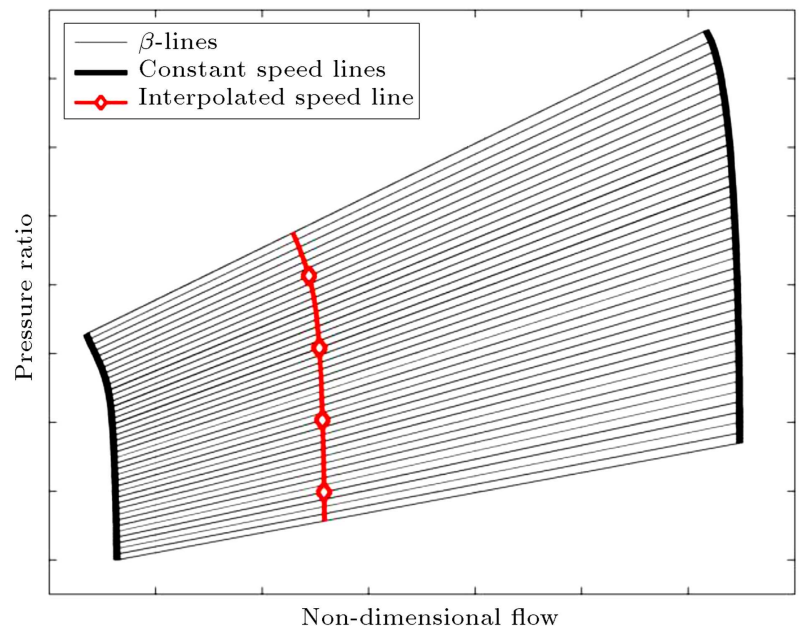

Figure 5. Speed line interpolation with $\beta$-lines method.

is obtained to determine the corresponding mass flow rate or isentropic efficiency. The interpolation in this step takes place between the points at the intersection of the red line and $\beta$-lines, shown in Figure 5 .

\section{Model tuning}

Simulations of the developed model have generally resulted in a rational behavior of the gas turbine; however, results inevitably do not exactly match the experimental data. Any mismatch may be due to:

- Model inefficacies in predicting complex phenomena such as combustion;

- Parametric errors, e.g., in cooling mass flow rate;

- Imprecise compressor and turbine characteristics;

- Manufacturing tolerances or gas turbine aging;

- Modeling simplifications.

To reduce such mismatches, eleven TPs are defined and included in the model. Ten TPs are applied to make a small shift in the characteristic curves of the compressor and four turbine stages. Five out of ten TPs are allocated to mass flow rates and the other five TPs to efficiency. An additional TP is used to correct the combustion efficiency. TPs are located in an additive way, and a zero vector is presumed as the nominal value.

As stated in Section 1, due to the lack of sensors, the estimation problem is underdetermined and has no unique solution. Considering a unique initial vector for the EKF, it would converge to a unique solution for an underdetermined estimation problem. The innovative methodology proposed in this paper is to seek the best solution by optimizing the EKF initial vector. Employing the EKF requires a linearized model of the gas turbine. Therefore, a linear state space model is derived from the presented nonlinear model via the linearize MATLAB function, which generates an LTI model of a nonlinear Simulink model at a given trim point as follows:

$$
\left\{\begin{array}{l}
\dot{x}=A x+B u+G w \\
y=C x+D u+L w
\end{array}\right.
$$

in which $x, u, y$, and $w$ represent the states, inputs, outputs, and TPs, respectively. This model is used to extract $\frac{\partial y}{\partial w}=-C A^{-1} G+L$ (in steady state) to be implemented in the EKF.

The EKF is used to minimize the mean squared error of the model, which is the average of the squares of the normalized errors of $1-g\left(u_{k}, w_{k}\right) / d_{k}$ by the use of sample pairs of the known input and the desired output, $\left(u_{k}, d_{k}\right)$, presuming the following representation of the gas turbine model:

$$
\left\{\begin{array}{l}
w_{k+1}=w_{k}+r_{k} \\
d_{k}=g\left(u_{k}, w_{k}\right)+e_{k}
\end{array}\right.
$$

where $g($.$) corresponds to the developed nonlinear$ model of the gas turbine and $r_{k}$ is the process noise defined as $r_{k} \sim N(0, Q)$. The general EKF algorithm for parameter estimation [21] can be applied as follows:

- The initial vector of TPs $\left(w_{0}\right)$ and the covariance matrix of TPs $\left(P_{0}\right)$ are estimated;

- The covariance matrices of artificial process noise and observation noise, i.e., $Q$ and $R$, are defined;

- In each step $1 \leq k \leq N$, TPs are updated using the following equations:

$$
\begin{aligned}
& P_{k}^{-}=P_{k-1}+Q \\
& K_{k}=P_{k}^{-} C_{k}^{w T}\left(C_{k}^{w} P_{k}^{-} C_{k}^{w T}+R\right)^{-1}, \\
& w_{k}=w_{k-1}+K_{k}\left(d-g\left(u_{k-1}, w_{k-1}\right)\right), \\
& P_{k}=\left(I-K_{k} C_{k}^{w}\right) P_{k}^{-},
\end{aligned}
$$

where:

$$
\left.C_{k}^{w} \triangleq \frac{\partial g\left(u_{k-1}, w\right)}{\partial w}\right|_{w=w_{k-1}}=-C A^{-1} G+L .
$$

The EKF algorithm is implemented iteratively in the proposed routine for optimizing the EKF initial vector, which includes the following steps:

1. Specify TPs to be tuned;

2. Generate an initial guess for the EKF initial vector $\left(w_{0}^{i}\right)$;

3. Carry out the EKF algorithm to obtain the corresponding vector of tuned $\operatorname{TPs}\left(w_{N}^{i}\right)$; 
4. Calculate the predefined modeling error (e.g., mean squared error):

a) If the modeling error is acceptable, return the optimal value of the EKF initial vector $\left(w_{0}^{*}\right)$ and the corresponding optimal tuned vector of TPs $\left(w^{*}\right)$

b) Else, update the EKF initial vector $\left(w_{0}^{i+1}\right)$ via the MATLAB lsqnonlin function and return to step 3;

By comparing the proposed tuning methodology with the presented scheme in [23], the following significant points are remarkable:

- The tuned values of TPs obtained by each underdetermined estimation method are not the same as the truth values;

- The time-consuming pseudo inverse calculation in [23] is eliminated in the proposed approach;

- Due to the implementation of the transformation matrix, $X$, in [23], the initial vector of the unknown parameters, $s$, should be defined in a way that the corresponding TPs are physically applicable in the model. Therefore, the initial vector of the unknown parameters should be a zero vector, and the TPs can only be defined in an additive way, while, in the proposed method, it is possible to define the TPs in either additive or multiplicative way;

- The EKF produces the tuned values of TPs as a local minimum around the initial vector of the EKF, which is assumed to be the optimization variable in the proposed methodology. Thus, based on the process knowledge, constraints could be imposed on the optimization of the EKF initial vector to obtain the optimal tuned values in a desired range. For example, the combustion chamber efficiency could be constrained to be less than its nominal value, which is ideally considered to be in the best possible situation. The proposed method in [23] does not have this capability since the unknown vector does not have a physical interpretation.

\section{Simulation results}

To study the behavior of the developed model, the SGT-600 twin-shaft gas turbine, introduced in Section 2, is considered as a case study. Its physical parameters and characteristics are embedded in the model and three different conditions are simulated to evaluate the proposed tuning procedure.

The first simulation involves modeling the behavior of the gas turbine under steady-state conditions in a range of ambient temperatures between $0^{\circ} \mathrm{C}$ to $30^{\circ} \mathrm{C}$. In this case, the tuning procedure is used using seven steady-state operating points associated with a step
Table 3. Main assumptions of the 1st simulation [33].

\begin{tabular}{lc}
\hline \multicolumn{1}{c}{ Parameter } & Value \\
\hline Ambient pressure $(\mathrm{kPa})$ & 101.325 \\
Output shaft speed (rpm) & 7700 \\
Inlet pressure loss (mbar) & 5 \\
Outlet pressure loss (mbar) & 5 \\
\hline
\end{tabular}

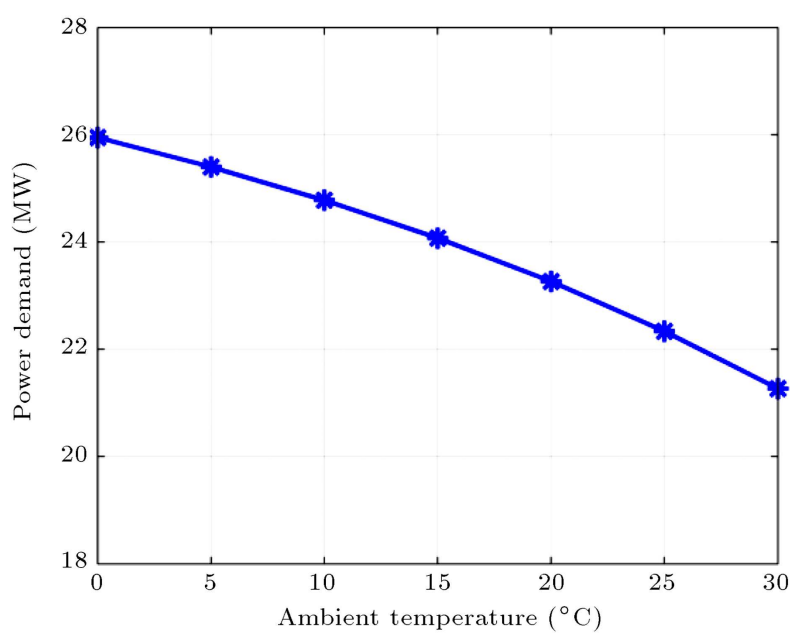

Figure 6. Output power demand set-point [33].

of $5^{\circ} \mathrm{C}$ at ambient temperature. Assumptions listed in Table 3 along with output power demand shown in Figure 6, which are declared in the gas turbine manufacturer's published data [33], are employed as model inputs. Only the measured datasets from three sensors including turbine outlet mass flow rate, heat rate, and turbine outlet temperature are used to tune eleven TPs. The simulation results of the tuned model in comparison with the manufacturer's data are depicted in Figure 7, in which acceptable conformity to a small mismatch is observed. The tuning procedure has thus reduced the modeling error from $9.52 \times 10^{-5}$ to $4.35 \times 10^{-10}$. Simulation results show that although the output power demand of the gas turbine model decreases at higher ambient temperatures, the turbine outlet temperature increases and the lower turbine outlet mass flow rate is required. Furthermore, the gas turbine efficiency drops by the ambient temperature rise, which is equivalent to the heat rate increment illustrated in Figure 7 . Since normalized errors are minimized in the tuning procedure, all the three outputs are tuned similarly, while a more accurate tuning of one specific output is achievable by including a multiplier weight larger than one for the corresponding output in the modeling error.

For the second case, the experimental dataset has been directly collected by the author under the supervision of Natural Iranian Gas Company (NIGC) 

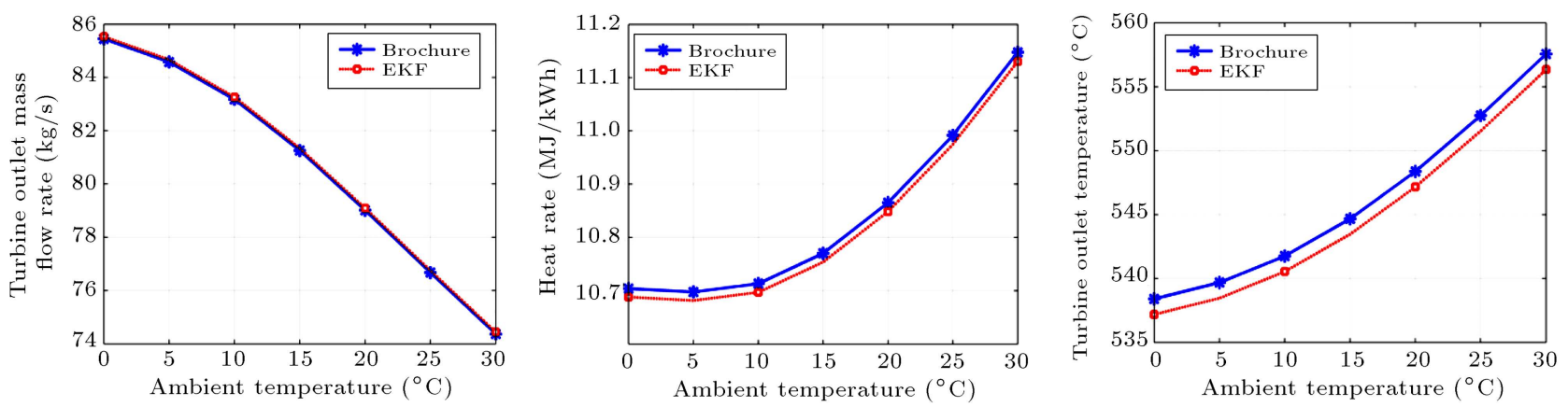

Figure 7. Simulation results versus ambient temperature for power demand changes (Figure 6) for SGT-600 employed as a turbo-generator in comparison with the manufacturer's published data.

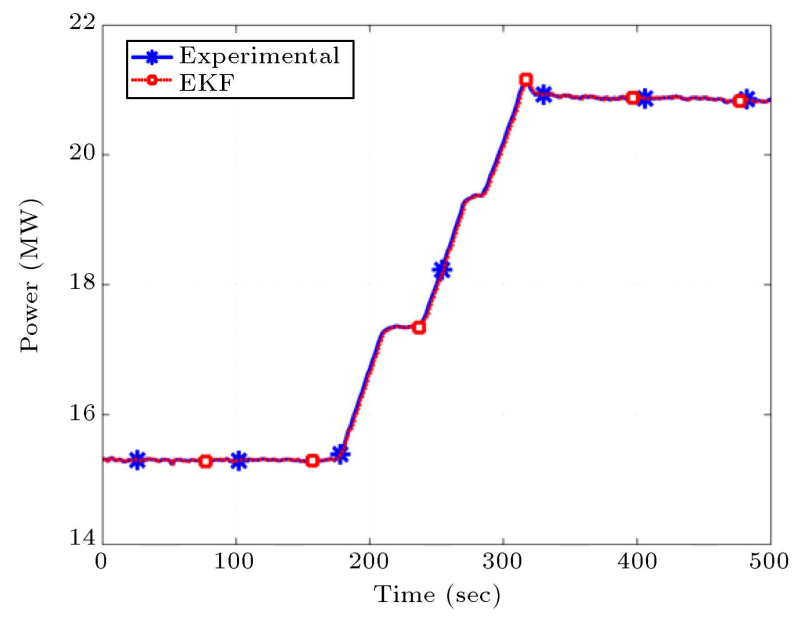

Figure 8. Output power demand set-point change. The experimental data have been collected by the author from a test rig located in Rey OCGT power plant [34].

along with Turbotec Corporation from a Test Rig located in Rey OCGT Power Plant [34] in IR Iran. This data has been extracted every two seconds from a practical test of the SGT-600 gas turbine as a turbogenerator, in which the output power demand changes from about 15.3 MW to 20.8 MW (Figure 8), while the output shaft speed is kept at $7737 \mathrm{rpm}$. Ambient temperature and pressure at site are about $19^{\circ} \mathrm{C}$ and $89 \mathrm{kPa}$, respectively. The model mismatch in two steady-state conditions shown in Figure 8 is minimized by the tuning procedure, by which the modeling error has reduced from $4.57 \times 10^{-4}$ to $1.01 \times 10^{-11}$. The simulation results in comparison with experimental data are depicted in Figure 9, in which the tuned gas turbine behavior follows the experimental data sufficiently well. Furthermore, the tuned values of TPs are listed in Table 4, almost all of which are too close to zero, showing that the nominal dynamic model is considerably accurate.

The other model tuning is performed based on the experimental data of the same gas turbine, i.e., SGT600 , used as a turbo-compressor in Neyzar natural gas compressor station [35] located near Qom in IR Iran.
Table 4. Tuned values of tuning parameters (2nd simulation).

\begin{tabular}{lc}
\hline \multicolumn{1}{c}{ Tuning parameters } & Tuned values \\
\hline Compressor mass flow & +0.041 \\
1st turbine stage mass flow & -0.034 \\
2nd turbine stage mass flow & -0.008 \\
3rd turbine stage mass flow & +0.002 \\
4th turbine stage mass flow & +0.003 \\
Compressor efficiency & +0.017 \\
1st turbine stage efficiency & +0.006 \\
2nd turbine stage efficiency & +0.015 \\
3rd turbine stage efficiency & +0.005 \\
4th turbine stage efficiency & +0.008 \\
Combustion chamber efficiency & -0.020 \\
\hline
\end{tabular}

The required dataset also has been directly collected by the author under the supervision of NIGC along with Turbotec Corporation. The power consumer, in this case, is a gas compressor that consumes the power as a function of its rotational speed and outlet to inlet pressure ratio. This function is extracted from the gas compressor characteristics and embedded in the model as the load module. The trend of changes in the gas compressor's rotational speed and pressure ratio is illustrated in Figure 10. Ambient temperature and pressure at site have been measured at about $27^{\circ} \mathrm{C}$ and $81 \mathrm{kPa}$, respectively. The experimental data have been recorded about every 6.24 seconds. Simulation results and experimental data are compared in Figure 11, in which an appropriate matching is obtained. In this case, minimizing the model mismatch error at three operating points has been targeted in the tuning procedure, in which the corresponding error is reduced from $7.68 \times 10^{-4}$ to $1.98 \times 10^{-11}$.

To report the average computation time per solution step of the model, an arbitrary simulation of SGT-600 is performed again on a PC featuring $3.10 \mathrm{GHz}$ Core i5 with $8 \mathrm{~GB}$ RAM, running windows 

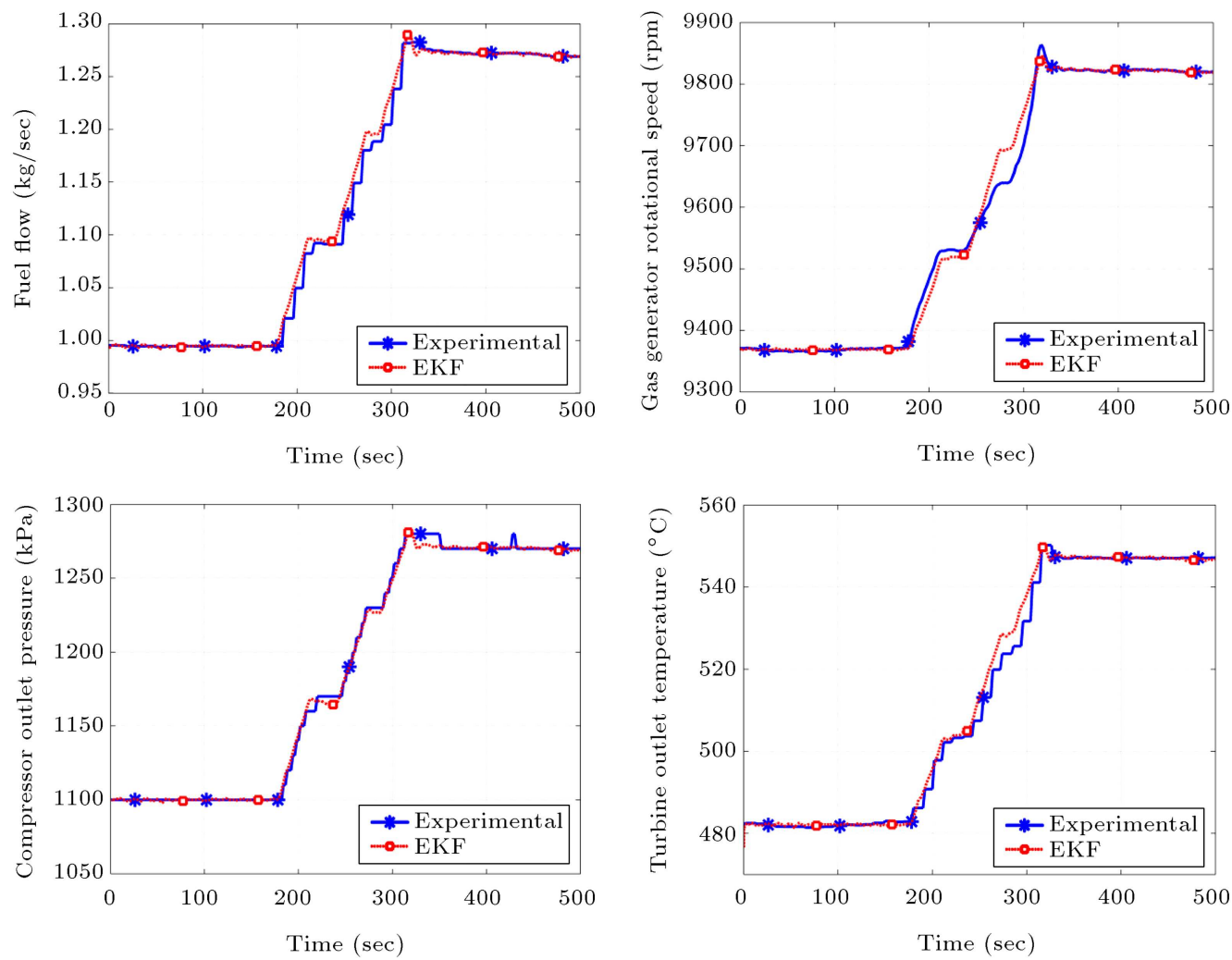

Figure 9. Comparison of the simulation and experimental results in response to load set-point change (Figure 8) for SGT-600 employed as a turbo-generator. The experimental data have been collected by the author from a test rig located in Rey OCGT power plant [34].
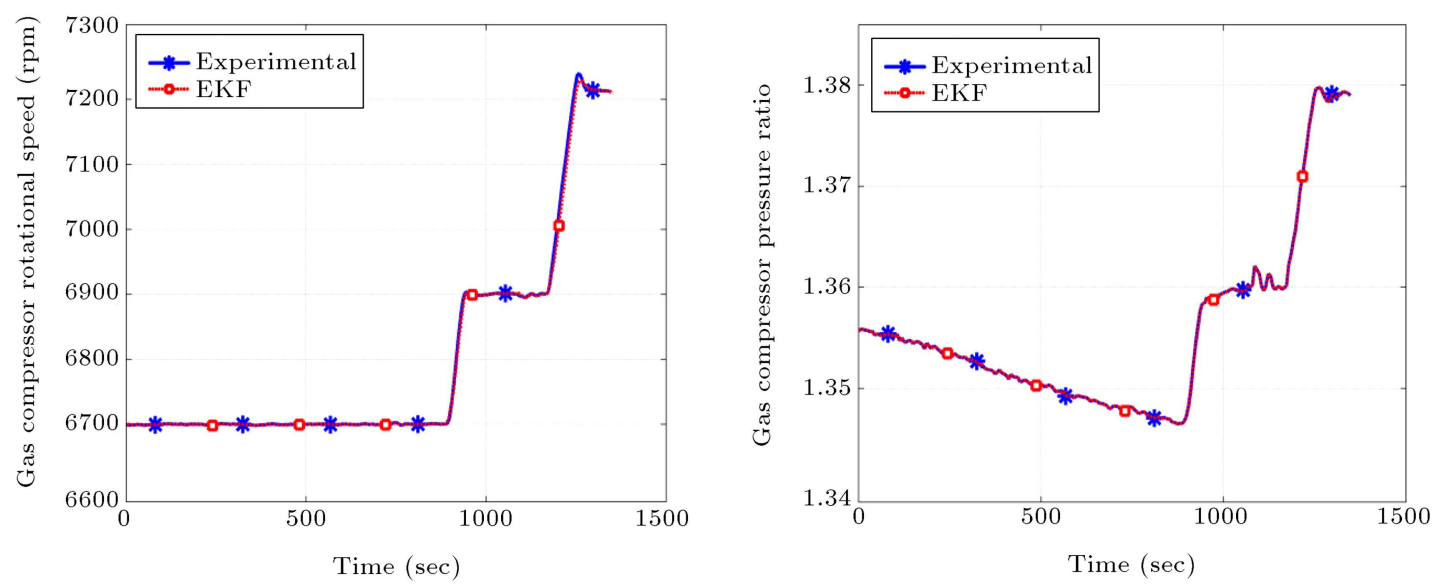

Figure 10. Trend of changes in the gas compressor's rotational speed and pressure ratio. The experimental data have been collected by the author from Neyzar natural gas compressor station [35].

7. The simulation step size is considered 0.02 second, which corresponds to the sampling period used by the governor of SGT-600. An average computation time per solution step of about $2.5 \mathrm{~ms}$ is obtained, which means that the model runs faster than what is essential for a real-time simulation. Therefore, $17.5 \mathrm{~ms}$ remains unused, which can be dedicated to a complex control algorithm, such as the model predictive control, to generate a more effective control signal.

\section{Conclusions}

A novel procedure to obtain a nonlinear dynamic model for simulating an arbitrary gas turbine engine as an object-oriented computer program was presented. The presented model is computationally efficient and makes it suitable to perform real-time simulations. Furthermore, its modular structure makes it highly intuitive and, thus, capable enough to simulate the 

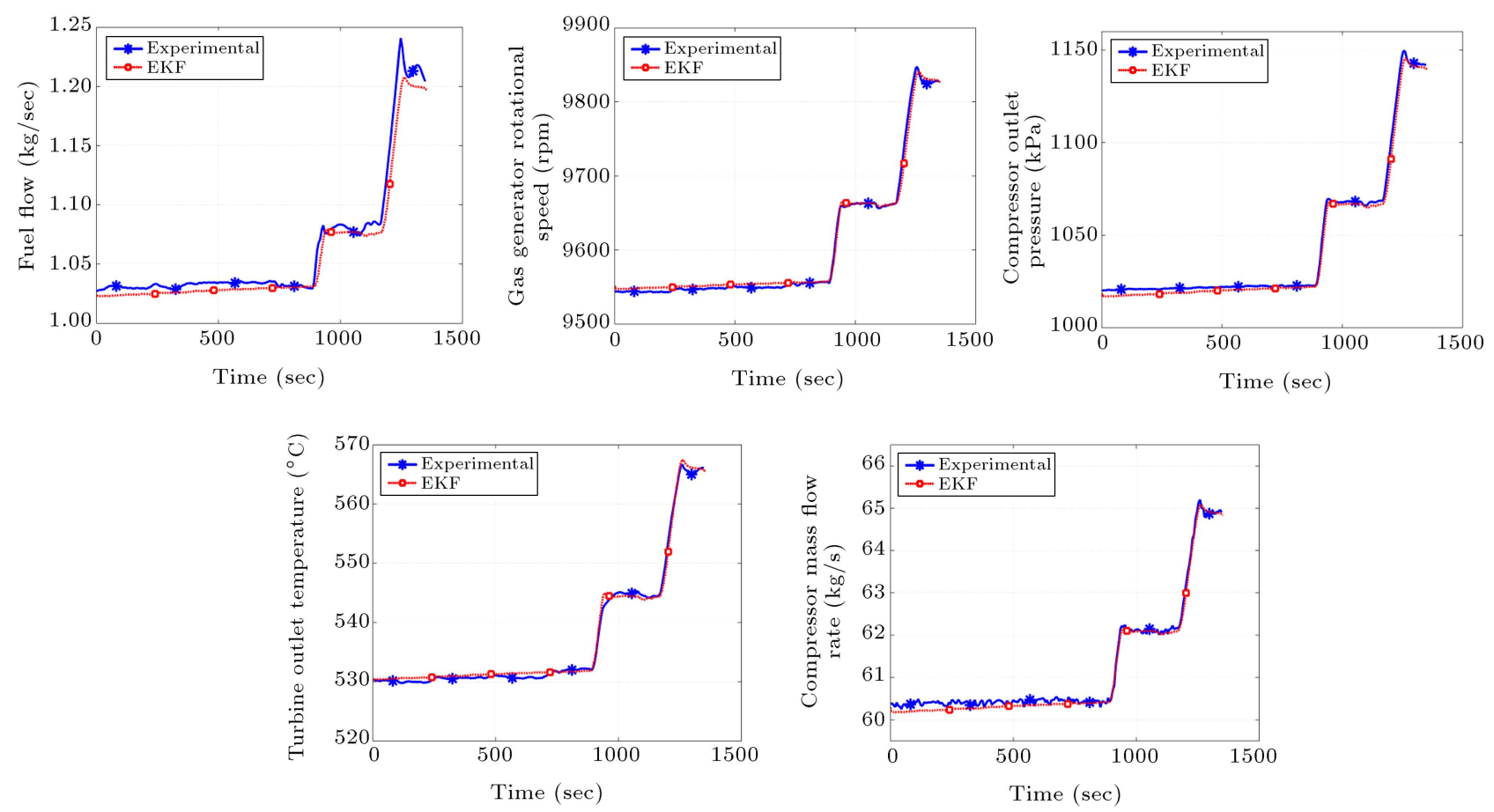

Figure 11. Comparison of the simulation and experimental results in response to load set-point change (Figure 10) for SGT-600 employed as a turbo-compressor. The experimental data have been collected by the author from Neyzar natural gas compressor station [35].

dynamic behavior of a great number of gas turbines by assembling different components with different arrangements. The introduced model is applicable to the following cases:

- Risk-free costless gas turbine simulation and performance study;

- Design, test, and tuning of gas turbine control systems;

- Fast noiseless data generation under various conditions;

- Faulty data generation by intentionally embedded faults;

- Obtaining linear local models by their linearization.

Afterwards, an innovative model tuning approach was introduced, in which an underdetermined estimation problem was solved on the basis of the EKF initial vector optimization. Suitable results were obtained using the proposed modeling approach along with the proposed model tuning scheme. To this end, the simulation of a twin shaft gas turbine under three different working conditions was studied. In each case, simulation results were compared with the experimental data that were gathered either from the manufacturer's published data or the gas turbines in service at sites.

\section{Nomenclature}

\section{Symbols}

$C_{p} \quad$ Specific heat at constant pressure $(\mathrm{J} / \mathrm{kg} \mathrm{K})$

$C_{v} \quad$ Specific heat at constant volume $(\mathrm{J} / \mathrm{kg}$ $\mathrm{K}$ )

$h \quad$ Total enthalpy $(\mathrm{J} / \mathrm{kg})$

$J \quad$ Moment of Inertia (kg.m²)

$m \quad$ Mass (kg)

$M \quad$ Molar mass $(\mathrm{g} / \mathrm{mol})$

$\dot{m} \quad$ Mass flow rate $(\mathrm{kg} / \mathrm{s})$

$P \quad$ Total pressure $(\mathrm{Pa})$

$P R \quad$ Pressure ratio

$q \quad$ Torque $(\mathrm{Nm})$

$R \quad$ Gas constant $(\mathrm{J} / \mathrm{kg} \mathrm{K})$

$t \quad$ Time (s)

$T \quad$ Total temperature (K)

$U \quad$ Internal energy $(\mathrm{J})$

$\nu \quad$ Volume $\left(\mathrm{m}^{3}\right)$

$V \quad$ Velocity $(\mathrm{m} / \mathrm{s})$

y Molar fraction

$\gamma \quad$ Specific heat ratio

$\eta \quad$ Isentropic efficiency 


$$
\begin{array}{ll}
\rho & \text { Density }\left(\mathrm{kg} / \mathrm{m}^{3}\right) \\
\omega & \text { Rotational speed }(\mathrm{rad} / \mathrm{s})
\end{array}
$$

\section{Subscripts}

$\begin{array}{ll}a & \text { Air } \\ c & \text { Compressor } \\ c c & \text { Combustion chamber } \\ \text { cool } & \text { Cooling flow } \\ f & \text { Fuel } \\ \text { in } & \text { Inlet } \\ l & \text { Load } \\ m & \text { Mechanical } \\ \text { out } & \text { Outlet } \\ p & \text { Plenum } \\ s & \text { Shaft } \\ t & \text { Turbine }\end{array}$

\section{Acknowledgment}

The authors would like to thank Natural Iranian Gas Company (NIGC) and Turbotec Corporation for their support of this research.

\section{References}

1. Cohen, H., Rogers, G.F.C., and Saravanamuttoo, H.I.H., Gas Turbine Theory, 4th Edn., Addison Wesley Longman, Harlow, UK (1996).

2. Evans, C., Rees, D., and Borrell, A. "Identification of aircraft gas turbine dynamics using frequency-domain techniques", J. Control Eng. Practice, 8(4), pp. 457467 (2000).

3. Asgari, H., Venturini, M., Chen, X., et al. "Modeling and simulation of the transient behavior of an industrial power plant gas turbine", ASME J. Eng. Gas Turbines Power, 136(6), pp. 061601-10 (2014).

4. Hosseini, S.M., Fatehi, A., Khaki Sedigh, A., et al. "Automatic model bank selection in multiple model identification of gas turbine dynamics", J. Syst. Control Eng., 227(5), pp. 482-494 (2013).

5. Asgari, H., Venturini, M., Chen, X., et al. "Modeling and simulation of the transient behavior of an industrial power plant gas turbine", ASME J. Eng. Gas Turbines Power, 136(6), p. 061601 (2014).

6. Asgari, H., Chen, X., Morini, M., et al. "NARX models for simulation of the start-up operation of a singleshaft gas turbine", Applied Thermal Engineering, 93, pp. 368-376 (2016).

7. Benyounes, A., Hafaifa, A., Kouzou, A., et al. "Gas turbine modeling using adaptive fuzzy neural network approach based on measured data classification", Mathematics-in-Industry Case Studies, 7(1), pp. 4-18 (2017).
8. Mohammadi, E. and Montazeri-Gh, M. "A new approach to the gray-box identification of wiener models with the application of gas turbine engine modeling", ASME J. Eng. Gas Turbines Power, 137(7), p. 071202 (2015).

9. Bahrami, S., Ghaffari, A., Sadati, S.H., et al. "Identifying a simplified model for heavy duty gas turbine", J. Mech. Science Tech., 28(6), pp. 2399-2408 (2014).

10. Razak, A.M., Industrial Gas Turbines, Performance and Operability, Woodhead Publishing, London, UK (2007).

11. Cao, Y., Jin, X., Meng, G., et al. "Computational modular model library of gas turbine", J. Adv. In Eng. Software, 36(2), pp. 127-134 (2005).

12. Schobeiri, T., Attia, M., and Lippke, C. "GETRAN: A generic, modularly structured computer code for simulation of dynamic behavior of aero-and power generation gas turbine engines", ASME J. Eng. Gas Turbines Power, 116(3), pp. 483-494 (1994).

13. Panov, V. "GasTurboLib: Simulink library for gas turbine engine modelling", In ASME Turbo Expo, Orlando, Florida USA, pp. 555-565 (2009).

14. Bianchi, M., Peretto, A., and Spina, P.R. "Modular dynamic model of multi-shaft gas turbine and validation test", In Proc. Winter Annual Meeting of ASME, 38, AES, Anaheim, Calif, USA, pp. 73-81 (1998).

15. Chacartegui, R., Sanchez, D., Munoz, A., et al. "Real time simulation of medium size gas turbines", $J$. Energy Conversion and Management, 52(1), pp. 713724 (2011).

16. Camporeale, S.M., Fortunato, B., and Mastrovito, M. "A modular code for real time dynamic simulation of gas turbines in Simulink", ASME J. Eng. Gas Turbines Power, 128(3), pp. 506-517 (2006).

17. Mohammadi, Z. and Salarieh, H. "Parameter identification of a parametrically excited rate micro-gyroscope using recursive least squares method", Scientia Iranica, Transaction B, Mechanical Engineering, 24(4), pp. 1889-1900 (2017).

18. Kiani, M. and Pourtakdoust, S.H. "Spacecraft attitude and system identification via marginal modified unscented Kalman filter utilizing the sun and calibrated three-axis-magnetometer sensors", Scientia Iranica, Transaction B, Mechanical Engineering, 21(4), pp. 1451-1460 (2014).

19. Panov, V. "Auto-tuning of real-time dynamic gas turbine models", In ASME Turbo Expo, Dusseldorf, Germany, p. V006T06A004 (2014).

20. Chaibakhsh, A. and Amirkhani, S. "A simulation model for transient behavior of heavy-duty gas turbines", Applied Thermal Engineering, 132, pp. 115127 (2018).

21. Simon, D., Optimal State Estimation, Kalman, $H \infty$, and Nonlinear Approaches, John Wiley \& Sons, Inc., Hoboken, NJ (2006). 
22. Litt, J.S. "An optimal orthogonal decomposition method for Kalman filter-based turbofan engine thrust estimation", ASME J. Eng. Gas Turbines Power, 130(1), 011601-1 (2008).

23. Simon, D.L. and Garg, S. "Optimal tuner selection for Kalman filter-based aircraft engine performance estimation", ASME J. Eng. Gas Turbines Power, 132(3), 031601-1 (2010).

24. Siemens, SGT-600 Industrial Gas Turbine, Siemens Industrial Turbomachinery, Inc., Duisburg, Germany (2005).

25. Strand, T., Industrial Gas Turbine Control, Siemens Power Generation, Siemens AG, Erlangen, Germany (2006).

26. Mackenzie, F.T., Our Changing Planet: An Introduction to Earth System Science and Global Environmental Change, 3rd Ed., Prentice Hall, NY, USA (2003).

27. Patai S. and Rappoport, Z., The Chemistry of Alkanes and Cycloalkanes, Wiley \& Sons, Chichester, UK (1992).

28. Kee, R.J., Rupley, F.M., and Miller, J.A., The Chemkin Thermodynamic Database, SAND87-8215B, UC-4, Sandia National Laboratories (1987).

29. Fielding, D. and Topps, J.E.C., Thermodynamic Data for the Calculation of Gas Turbine Performance, Aeronautical Research Council, R\&M, No. 3099 (1959).

30. El-Masri, M.A. "GASCAN-An interactive code for thermal analysis of gas turbine systems", ASME J. Eng. Gas Turbines Power, 110(2), pp. 201-209 (1988).

31. Tabari, A., Khaledi, H., and Hajilouy Benisi, A. "Comparative evaluation of advanced gas turbine cycles with modified blade cooling models", In ASME Turbo Expo, Barcelona, Spain, pp. 537-546 (2006).

32. Rowen, W. "Simplified mathematical representations of heavy duty gas turbines", ASME J. Eng. Gas Turbines Power, 105(4), pp. 865-869 (1983).

33. SGT-600 Industrial gas turbine (power generation) published data, accessed at 2018, July 22, Retrieved from http://www.energy.siemens.com/ru/pool/hq/ power-generation/ gas-turbines/SGT-600/downloads / SGT-600_GT_PowerGen_EN.pdf

34. Rey OCGT Power Plant, accessed at 2018 July 22, Retrieved from http://globalenergyobservatory. org/geoid/45024
35. Neyzar Natural Gas Compressor Station, accessed at 2018 July 22, Retrieved from http://tcproject. nigc.ir/Portal/Home/ShowPage.aspx?Object=NEWS \&CategoryID=21dff668-6bec-4fd1-8430-81a194b7d9e2 \&WebPartID=893aaf0b-0d62-4c3b-82bf-55098c09e804 $\& I D=47 a 415 c 7-908 a-4297-b a 13-c b 8 c 7 c a 9 c a 8 c$

\section{Biographies}

Reza Sheikhbahaei received his MS degree in Mechanical engineering from Sharif University of Technology (SUT), Tehran, Iran in 2012. He is currently working towards a $\mathrm{PhD}$ degree at Sharif University of Technology, Tehran, Iran. His research interests include applying explicit model predictive control, robust control, fault-tolerant control, and mechanical systems identification.

Gholamreza Vossoughi received his $\mathrm{PhD}$ from Mechanical Engineering Department at the University of Minnesota in 1992. Ever since, he has been a faculty member at Mechanical Engineering at Sharif University of Technology, Tehran, Iran. He has served as the Director of Manufacturing Engineering and Applied Mechanics Division, Graduate Dean and Chairman of Mechanical Engineering Department at Sharif University of Technology. His research interests include bioinspired robotics, micro-robotic systems, man-machine interface and haptic systems, mechatronics, and control systems. He has published more than 90 peer-reviewed journal papers in these fields.

Aria Alasty received his $\mathrm{BSc}$ and $\mathrm{MSc}$ degrees in Mechanical Engineering from Sharif University of Technology (SUT), Tehran, Iran in 1987 and 1989. He also received his $\mathrm{PhD}$ degree in Mechanical Engineering from Carleton University, Ottawa, Canada in 1996. At present, he is a Professor of Mechanical Engineering at Sharif University of Technology, Tehran, Iran. He has been a member of Center of Excellence in Design, Robotics, and Automation (CEDRA) since 2001. His fields of research are mainly in nonlinear and chaotic systems control, computational nano/micro mechanics and control, special purpose robotics, robotic swarm control, and fuzzy system control. 